\title{
Research on Position Detection Algorithm of Switched Reluctance Motor Based on FPGA
}

\author{
Xu Gang \\ Zhonghuan Information College Tianjin University of Technology, No. 99 Liu Kou Road, Xiqing District, Tianjin
}

\begin{abstract}
In this paper, the Cyclone series programmable logic device (FPGA) is used to implement the location detection algorithm. The implementation process requires that the storage space be occupied as small as possible, fast, and less occupied by CPU resources. High speed A/D is used for signal detection conversion. Pipeline optimization technology is adopted to improve working speed. Program module and top layer file is compiled. Experimental simulation verifies the effectiveness and real time of the algorithm.
\end{abstract}

\section{INTRODUCTION}

In the current practical application, the position information is usually obtained by axis position sensor or other type of location detector, it not only improves the cost and complexity of the system, but also reduces the robustness of the structure of the SRD system and affects the reliable operation of the whole system. Therefore, the position detector is removed and the rotor position is indirectly determined by using the voltage and current information of the motor, so that the structure of the system is more solid, and the operation is more reliable, efficient, and the cost is lower, which is undoubtedly a potential research direction.

FPGA has a unique advantage to realize the hardware platform of indirect detection. In the algorithm aspect, it not only solves the shortcoming of the custom circuit, but also overcomes the shortcomings of the original programmable gate circuit number. Therefore, it can be used to achieve high speed algorithm control, both from the programming scale and the speed of work.

\section{SIMPLIFIED MAGNETIC CHAIN METHOD}

\subsection{The Principle Of Simplified Magnetic Chain Method}

The simplified flux linkage method is proposed under the condition of single-phase that turns conduction and voltage PWM control in order to improve the real-time performance of the algorithm, extend the applicable speed range and reduce the required memory. The basic idea of the simplified magnetic chain method: when the single phase of the motor is turned on, the information of each position of the rotor is not needed, it is able to judge whether it has reached the position of the commutation, the rotor position detection can be simplified to the detection of commutation position. The value of the estimated magnetic chain obtained by the integral calculation is compared with the value of the reference flux linkage corresponding to the commutation position of the current, if the former is larger than the latter, it is considered that the position of the commutation has arrived, the current phase is turned off, and the next phase is guided, on the other hand, it is considered that the position of the commutation is not reached, and the current phase is continued(A.V Radun 1998).

The position of the commutation is generally near the maximum position of the inductor, the shape of the magnetic chain current--curve corresponding to the different inductors is similar. Therefore, we only need to test and store the flux linkage current curve at the location of maximum inductance. First, the reference magnetic chain is checked from current to the position of the maximum inductor, and the reference flux value of the corresponding position of the commutation position is obtained. The algorithm only needs to establish the magnetic chain - current curve of the maximum inductance position, then the two - dimensional table is queried. The algorithm needs small memory, simple and fast, the test results are more accurate and reliable.

\subsection{The Realization of Simplified Magnetic Chain Method}

Because each phase of the motor is completely symmetrical, the magnetic chain of any phase of the motor current--curve can be measured(U.S Deshpand 2015). The calculation of magnetic chain is used for trapezoid method. The current and voltage sampled by current and voltage sensors are converted by high speed

*Corresponding author: xugang19861986@126.com 
$\mathrm{A} / \mathrm{D}$, the real-time magnetic chain of each sampling point is calculated.

If duty cycle of chopper cycle is $\tau$, sampling the current value ik is unchanged in the $\mathrm{K}$ period, voltage is supply voltage uk in the opening period of $\tau T$, The freewheeling chopper voltage is zero in the zero voltage period $(1-\tau) \mathrm{T}$. If the winding resistance $\mathrm{R}$ is constant, it is not changed with time, the flux linkage formula at the current sampling time can be obtained:

$$
\psi(k)=\psi(k-1)+\Delta \psi(k)
$$

The calculated values of each $\psi(\mathrm{k})$ is compared to the measured reference magnetic chain, when the current person is larger than the latter, it turn off the current phase and guide the next phase; on the other hand, it continue to guide the current phase.

\section{SYSTEM HARDWARE DESIGN}

The system block diagram of a position sensorless phase is obtained by a simplified magnetic chain method.

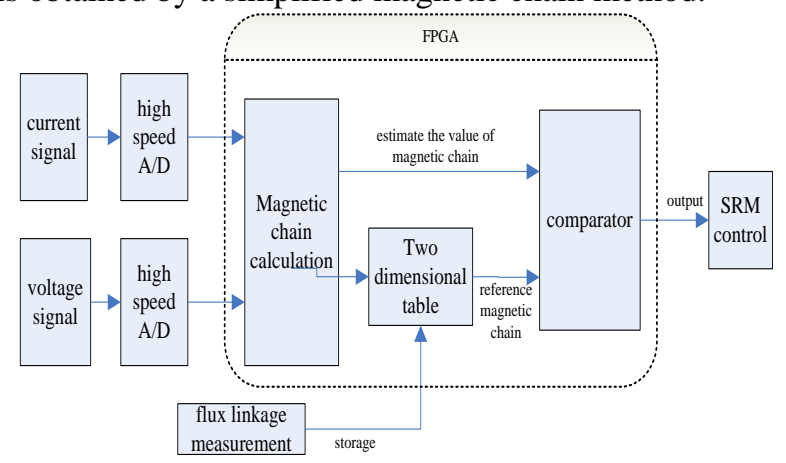

Figure 1: Current-flux linkage curve

TLC5510 chip is used in high speed A/D, TLC5510 is a new analog to digital converter (ADC) produced by the TI company in the United States. Because of the half flash structure and the CMOS technology, the number of comparators is greatly reduced, and at the same time, the low power consumption can be maintained at high speed. Because TLC5510 has not only high speed A/D conversion function, but also with internal sampling circuit, which greatly simplifies the design of peripheral circuit; at the same time, because with the internal standard resistor, it can obtain the reference voltage $2 \mathrm{~V}$ full scale from the $+5 \mathrm{~V}$ power supply.

\section{SYSTEM SOFTWARE DESIGN}

The control strategy of the system is based on the calculation of the magnetic chain and the query two-dimensional table. Through the phase current is converted into digital signal and processed in FPGA, when the detected value is greater than the estimated flux flux reference value, turn off the current phase and next phase conduction(T J E Miller 2002), so as to realize the control of the SRM. The flow chart of the overall software control algorithm is shown as shown in the diagram.

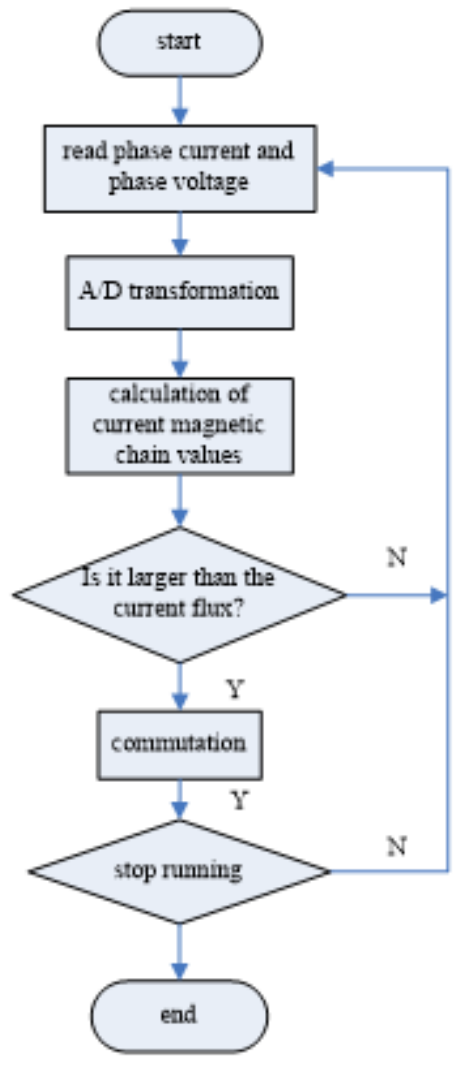

Figure 2: Arithmetic flow chart

At the top of the program, the software has the initialization program and the main program. After the necessary initialization, the main program begins to execute. The following focuses on the software design of the main program.

\subsection{Current Sampling}

According to the Shannon sampling theorem, the sampling frequency must meet the requirement of more than or equal to two times the frequency of the sampled signal.

$$
f_{s} \geq 2 f
$$

The frequency of the signal to be sampled in this system is the change frequency of the phase current I in the switched reluctance motor. The test prototype used in this system is a $8 / 6$ pole four phase switched reluctance motor, its main parameters are as follows: rated power $0.375 \mathrm{KW}$, rated voltage $220 \mathrm{~V}$ (DC side), rated speed $1500 \mathrm{r} / \mathrm{min}$. According to the above parameters and the operating principle of the switched reluctance motor, we can get the following corresponding relation:

$$
\frac{n}{60}=\frac{f_{\text {in }}}{6}
$$

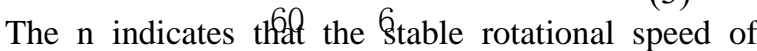
switched reluctance motor, unit is $\mathrm{rad} / \mathrm{min}$. fin indicates the phase current frequency of the switched reluctance motor (SRM) phase $\mathrm{N}$ phase, unit is $\mathrm{Hz}$.

According to the parameter $\mathrm{n}=1500 \mathrm{rad} / \mathrm{min}$, the phase current fundamental frequency of the corresponding switched reluctance motor can be obtained. 


$$
f_{\text {in }}=\frac{1500}{60} \times 6=150 \mathrm{~Hz}
$$

Therefore, the high speed A/D converter can be realized from the programming scale and the speed of work. There are enough for data processing, calculation, or waveform reduction about $4000 / 150=26$ sampling points in a cycle.

\subsection{Create a Current - reference Magnetic Chain Data Form}

The maximum inductor position of each current is measured for simplified magnetic chain method. Table 1 lists the current and magnetic chain data for the maximum inductance.

Table 1: Flux linkage data at the largest inductance

\begin{tabular}{|c|c|c|c|c|c|c|c|}
\hline$I(\mathrm{~A})$ & 2 & 4 & 6 & 8 & 10 & 12 & 14 \\
\hline$\psi(\mathrm{Wb})$ & 0.3769 & 0.6878 & 0.8120 & 0.8742 & 0.9171 & 0.9468 & 0.9661 \\
\hline
\end{tabular}

the output port corresponds to the reference value of the flux linkage. This process can be verified by the simulation waveform. Figure 3 shows the simulation waveform of the reference flux data memory.

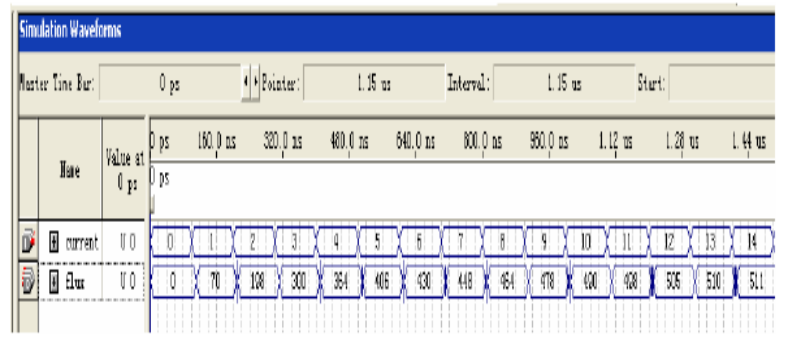

Figure 3: Current-flux linkage data storage

\subsection{Determine the Commutation Position}

The current value of the flux linkage is compared with the reference flux value pre measured and stored. The output is used as a control signal to directly control the switched reluctance motor. When the former is less than the latter, the output is "0", the motor does not commutate, otherwise, the output is "1", and the motor commutation.

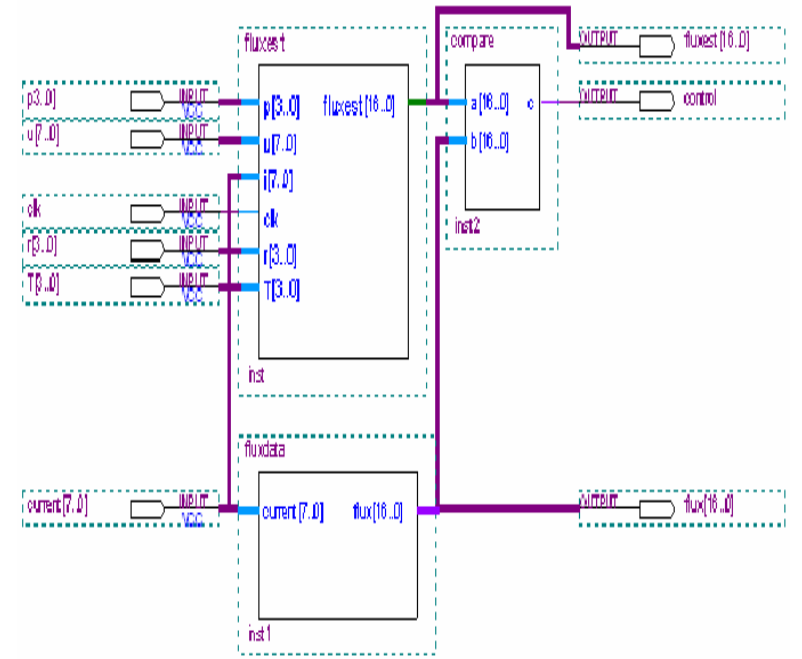

Figure 4: Phase changing position control scheme

Simulation results show that the output of the control signal can accurately reflect the relationship between the current flux value and the reference flux value, so as to control the commutation of switched reluctance motor.

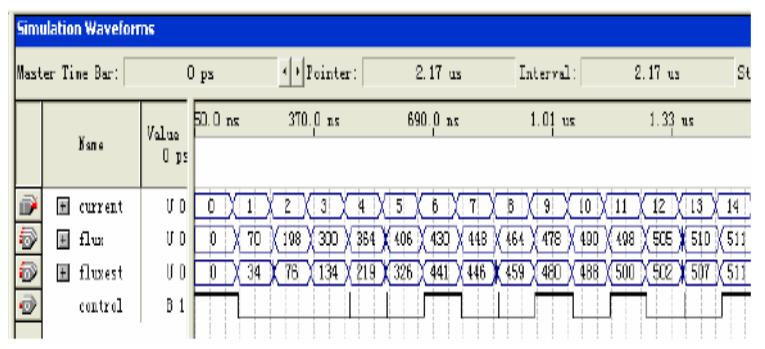

Figure 5: Simulation result of Phase changing control

\section{CONCLUSION}

In this paper, the data acquisition of current and voltage is realized by high speed $\mathrm{A} / \mathrm{D}$, and the conversion rate of data is up to $20 \mathrm{Msps}$, which meets the requirement of fast data conversion. The trapezoidal integral form is used to calculate the current flux value of the motor, and compared with the measured flux linkage data stored in $\mathrm{ROM}$, the rotor position is determined, then the location of commutation point is identified. The effectiveness and real-time performance of the algorithm is verified through the simulation of the sub modules and the top level files based on FPGA technology.

\section{REFERENCES}

1. A.V Radun. High power density switched reluctance motor applications drive for aerospace. IEEE-IAS Annu.Metting(C). 1998, pp. 568-573.

2. U.S Deshpand. High-force density linear switched reluctance machine. IEEE Trans. on IA(C). 2015, 31(5). 345-352.

3. T J E Miller. Optimal design of switched reluctance motors. IEEE Trans on industrial electronics(C). 2002, vo1 49 Nol, 15-27. 